\title{
Infancia en Chile I973-20I3: 40 años de tensiones e inflexiones neoliberales
}

\author{
Paula Cubillos-Celis, Ph. D. ${ }^{a}$ \\ Agencia Francesa de Desarrollo, \\ Université Paris Descartes, Francia
}

\section{Resumen (analítico)}

El objetivo del artículo es analizar las formas que adopta el Estado social en Chile entre 1973-2013, mediante una lectura de las políticas de derechos de la infancia y las tensiones que se desprenden de dicho proceso. El principal resultado del trabajo es que las transformaciones observadas introducirán tres inflexiones a la estructura de distribución de derechos: liberalización, tecnocratización y focalización. Las políticas de salud, educación y protección especializada reflejarán tanto el modelo de continuidad como las inflexiones neoliberales, y representarán un terreno privilegiado de inteligibilidad del proceso sociopolítico y económico. A través de una metodología cualitativa de análisis de entrevistas, del marco legal y de la documentación sectorial, esta investigación propone retrasar la reconfiguración de lo social en Chile desde el rostro de la infancia durante el proceso de neoliberalización.

\section{Palabras clave}

Estado social, políticas sociales, infancia, neoliberalismo, Chile.

\section{Thesauro}

Tesauro de Ciencias Sociales de la Unesco.

\section{Para citar este artículo}

Cubillos-Celis, P. (2021). Infancia en Chile 1973-2013: 40 años de tensiones e inflexiones neoliberales. Revista Latinoamericana de Ciencias Sociales, Niñez y Juventud, 19(3), 1-22.

https://dx.doi.org/10.11600/rlcsnj.19.3.4868

\section{Historial}

Recibido: 18.01.2021

Aceptado: 15.04 .2021

Publicado: 25.08.2021

\section{Información artículo}

El artículo presenta resultados de la investigación titulada L'État social au Chili sous le prisme des politiques de l'enfance: continuités et inflexions 1973-2013, realizada para la obtención de grado de Doctora en Sociología de la Universidad Paris Descartes-Francia, y financiada por la Beca Chile-Conicyt. Investigación realizada entre el 01/10/2013 y el 26/09/2017. Área: Ciencias Sociales. Subárea: Sociología. 


\section{Childhood in Chile 1973-2013: 40 years of neoliberal tensions and inflections}

\section{Abstract (analytical)}

The objective of this article is to analyze the different approaches adopted by the Chilean social welfare system towards child protection between 1973 and 2013, and to analyze the tensions that emerged from the process of neo-liberalization that occurred during this period. The main result of this work is that the transformations observed since 1973 involved three processes that affected the structure of rights distribution: liberalization, technocratization and targeting. Health, education and specialized protection policies reflect both the continuity model and neoliberal influence, and therefore offer a privileged standpoint for analyzing the socio-political and economic processes that occurred in Chile during this period. Through a qualitative methodology involving analysis of interviews, the legal framework and sectoral documentation, this research reflects on 40 years of the reconfiguration of social services in Chile and the effects on childhood during the process of neo-liberalization.

Keywords

Welfare State, social policy, childhood, neoliberalism, Chile.

\section{A infância no Chile I973-20I3: 40 anos de neoliberal tensões e inflexões neoliberais}

\section{Resumo (analítico)}

O objetivo deste artigo é analisar as formas adotadas pelo Estado social no Chile entre 1973 e 2013, com base na proteção dos direitos da criança e analisar as tensões que surgem do processo de neoliberalização. O principal resultado deste trabalho é que as transformações observadas desde 1973 irão introduzir três inflexões na estrutura da distribuição de direitos: liberalização, tecnocratização e focalização.. As políticas de saúde, educação e proteção especializada espelham tanto o modelo de continuidade, quanto as inflexões neoliberais e serão apresentadas como um terreno privilegiado de inteligibilidade do processo sócio-político e económico vivido pelo país. Através de uma metodologia qualitativa de análise de entrevistas, do enquadramento legal e da documentação sectorial, esta investigação propõe adiar 40 anos da reconfiguração do social no Chile, da face da infância durante o processo de neoliberalização.

\section{Palavras-chave}

Estado de bem-estar, política social, infância, neoliberamismo, Chile.

\section{Información autores}

[a] Doctora en Sociología, Universidad Paris Descartes-USPC. Magíster en Ciencias Sociales mención Estudios Políticos, Ecole des Hautes Etudes en Sciences Sociales Ehess-Paris. Magíster en Ciencia Política FlacsoEcuador. Magíster en Antropología y Desarrollo, Universidad de Chile. Investigadora Asociada al Laboratorio Cerlis, Francia, de la Universidad Paris Descartes-Universidad Paris Sorbonne Cité (USPC), la Universidad Sorbonne Nouvelle y el CNRS (Centro Nacional de Investigación Científica). iD 0000-0001-8716-0485. H5: 2. Correo electrónico: cubilloscelisp@afd.fr 


\section{Introducción}

esde la década de los setenta, Chile vive un conjunto de transformaciones en
los ámbitos económico, político y social, dando paso a un proceso de reconfiguración de la concepción del Estado, la solidaridad y los derechos sociales. Diversas reformas sociales harán eco a las reformas económicas realizadas a partir de 1973, año de inicio de la dictadura militar que derrocó al gobierno del Presidente Salvador Allende (1970-1973); estas tendrán por objetivo dar una nueva forma al Estado social, a partir del proceso de neoliberalización económica. Las diversas olas de reformas que tendrán lugar entre 1973 y 2013 se transformarán en instrumento de modelización: de la nueva matriz sociopolítica de la dictadura (1973-1989), de las negociaciones del proceso democratizador durante el periodo de transición (1990-1999) y del mecanismo de legitimación democrática del modelo económico durante el periodo de consolidación (2000-2010).

Desde esta perspectiva, nuestra hipótesis es que, en la continuidad de la historia social y de una estructura de distribución desigual del acceso a los derechos (que sostiene al Estado social desde el nacimiento de la República hasta los años setenta), la reestructuración neoliberal impone sus propias inflexiones en la relación entre el Estado social y los individuos, marcada por una despolitización sin precedentes de las cuestiones sociales (Cubillos-Celis, 2019a). El modelo de distribución desigual de derechos se ve aumentado en su campo de atribuciones, a través de la implementación del Estado residual ${ }^{1}$ en dictadura y, luego, progresivamente durante la recuperación y consolidación de la democracia, tomando forma en las políticas de infancia. Las inflexiones que intervienen son la liberalización de los sectores sociales, la tecnocratización de las políticas sociales y la implementación de la focalización (Cubillos-Celis, 2019b).

\footnotetext{
${ }^{1}$ La noción de Estado social residual hace alusión al concepto propuesto par Titmuss (1974) que entiende al mercado como el único agente corrector de los problemas sociales. El Estado jugará un rol subsidiario. Esta noción será retomada después por Esping-Andersen (2009) para caracterizar los regímenes de bienestar liberales.
} 
La introducción de dichos ejes estructurantes de lo social se cristaliza en la primera etapa del proceso de neoliberalización (1973-1989) a través del denominado Plan de siete modernizaciones (implementado desde 1979) y que comprende, entre otros, los sectores de la educación, la salud y la justicia. Las reformas de las políticas de la infancia, ${ }^{2}$ particularmente los sistemas de educación, de salud primaria y de protección especial, serán el reflejo de un Estado social bajo tensión entre el rol fuertemente regulador que acompaña la liberalización de los sectores sociales y la apropiación progresiva de un discurso sobre los derechos sociales, que hace eco de los procesos de democratización vividos por el país desde 1990 .

En este marco, las políticas de infancia constituyen un nicho histórico de intervención que permite analizar la acción pública en perspectiva e identificar un campo de acción continua. El interés político por los asuntos de la infancia ha llevado al Estado chileno a efectuar un gasto social considerable desde la dictadura, a pesar de la filosofía residual practicada por el régimen, surgiendo la pregunta: ¿por qué la infancia es un eje central de inversión pública en un momento en que se reduce la intervención directa del Estado?

Este interés se puede explicar en función de aspectos psicológicos, sociológicos y económicos. Desde los argumentos psicológicos, los primeros años de la vida de una persona son concebidos fundamentales para el desarrollo de sus habilidades emocionales, cognitivas y sociales. En lo que respecta a la sociología, el interés por la infancia consiste en identificar una categoría social que va más allá de un grupo de edad y que es determinante en el despliegue de estrategias de desarrollo social que permitan reducir la pobreza y la desigualdad. El componente económico asocia a la infancia con el potencial de rentabilidad de las inversiones, posteriormente visibles en el mercado de trabajo.

Al descifrar el significado de la acción del Estado de Chile hacia los niños y niñas como categoría objetivo, durante el período 1973-2013, pretendemos comprender qué ha cambiado socialmente desde la instalación del neoliberalismo. ¿Cómo se reposiciona el Estado social y cómo se rearticula lo social sobre la base de las transformaciones político-económicas?

Este interés particular surge dado que, aunque diversas investigaciones han abordado los cambios en la política de protección de la infancia de Chile, la mayoría de ellas

\footnotetext{
${ }^{2}$ En esta investigación, la categoría infancia es abordada desde una dimensión minimalista y operacional en referencia a la edad de vida, que determina su inclusión en las políticas sociales en Chile (desde los o a los 12 años).
} 
centran su atención en los grados de realización de los derechos económicos, sociales y culturales de los niños y las niñas, mientras que nuestro interés se dirige hacia una lectura del fenómeno de la transformación del Estado social a través de las políticas sociales de la infancia.

En este contexto, la pregunta sobre la concepción del neoliberalismo es un tema clave, sobretodo porque dicho debate ha sido ampliamente trivializado y la resistencia a los procesos de financiarización y la globalización de la economía han producido un efecto de indistinción entre la dinámica del propio mercado y la forma específica del neoliberalismo en el lenguaje común (Therborn, como es citado en Cubillos-Celis et al., 2019). Sin embargo, una identificación precisa de los procesos de neoliberalización y de las formas que adoptan los Estados sociales es un paso necesario para hacer entender los procesos e identificar y comprender las reconfiguraciones sociales propias de las dinámicas en curso.

Esta investigación aborda la cuestión del neoliberalismo en Chile como un modelo de desarrollo que remplaza el modelo de sustitución de importaciones vigente hasta 1973, y examina el fenómeno de neoliberalización de la sociedad chilena. Como hecho fundador de dicho proceso, observamos que el proyecto de capitalismo centralizado representado por Allende se percibe como una ruptura del histórico proyecto republicano liberal y del pacto mesocrático. La dictadura proclama, así, restaurar el orden y reconstruir el proyecto histórico bajo la égida de los militares.

En este sentido, el modelo aplicado no es solo un modelo de desarrollo entre otros, sino un proyecto de restitución del poder de clase de las élites (Harvey, 2005), que elimina los espacios de mediación entre los distintos sectores sociales. Desde este punto de vista, la tendencia al socialismo habría causado una ruptura en la evolución político-económica de la civilización occidental; evolución que busca maximizar las fuerzas sociales espontáneas de los individuos y alejarlas de la coacción impuesta por el Estado (Hayek, 2014). Sin embargo, la propuesta neoliberal se aleja de las concepciones liberales clásicas, proponiendo abordar la crisis de gobernanza del modelo clásico de Manchester y generar respuesta a los problemas de la ideología naturalista del laissez-faire (Dardot \& Laval, 2010; Harvey, 2005). Por ello, el Coloquio Walter Lippmann (París, 1938) y la fundación de la Sociedad Mont Pèlerin (Suiza, 1947) se propusieron repensar la lógica de la gobernanza del capitalismo competitivo, proponiendo la necesidad de una vigilancia y regulación constantes a través de métodos que puedan orientar la actividad económica más allá del laissez-faire (Dardot \& Laval, 2010). En otras palabras, la competencia como principio de organización social requeriría ciertos tipos de acción gubernamental (Hayek, 2014), lo 
que implica repensar la relación entre el mercado y la sociedad, así como entre el Estado y los individuos; y, de tal manera, instalar una nueva racionalidad en la que el Estado sea un facilitador de este mecanismo.

En esta perspectiva, el proceso de reforma llevado a cabo en Chile a partir de 1973 -luego del golpe de Estado- busca restaurar el poder de clase y dar forma al neoliberalismo como una filosofía de articulación de la vida social, política y económica, más que como un modelo de conducta económica ortodoxo.

Esto nos lleva a abordar los debates en torno a la concepción del bienestar y del Estado social. En dicho registro, consideramos que la noción de regímenes de bienestar propuesta por Esping-Andersen (2009), ampliamente utilizada en el contexto europeo, se aleja de la realidad latinoamericana, tanto por su diversidad como por los procesos asimétricos que presiden la configuración de las instituciones. En América Latina, los sistemas de protección distribuyen asimétricamente la responsabilidad entre los diferentes actores involucrados (el Estado, las élites, las comunidades y el mercado), y las formas institucionales de protección se caracterizan por la ausencia de mercados laborales formales y economías industrializadas. Tomando dichas consideraciones, los sistemas de bienestar propuestos tanto por Titmuss (1974) como por Esping-Andersen intervienen en esta investigación como mapas cognitivos que permiten entender dichos fenómenos como tendencias y como resultados sociohistóricos específicos.

El desarrollo de un régimen de organización de la protección en Chile es entendido como un proceso de modelos mixtos. La estructura histórica de la distribución del acceso a los derechos propuesta posee características de los regímenes de beneficios estratificados descritos por Filgueira (2007), así como características de los regímenes conservadores propuestos por Esping-Andersen. A esto se añadirá la complejidad de la reconfiguración del Estado social bajo las dinámicas de reestructuración neoliberal, la cual integra la relación entre el desarrollo de los Estados y el mercado mundial, los intereses financieros, las instituciones, ideas, ideologías, así como las influencias internacionales (Gough \& Therborn, 2010).

La recomposición de los regímenes de bienestar a nivel regional desde la década de los años ochenta pone en cuestión las clasificaciones de los modelos clásicos del bienestar; por ello nos proponemos contribuir a descifrar los significados que recorren la configuración neoliberal de la filosofía de la protección a partir de una lectura de la experiencia chilena de 1973 a 2013. A la luz de esta interpretación, el objetivo de esta investigación es analizar las formas que adopta el Estado social a partir de la restructuración político- 
económica del país y, específicamente, descifrar la forma en que el Estado social en Chile concibe y opera las políticas de bienestar infantil durante 40 años (1973-2013), analizando los cambios, continuidades y rupturas en las distintas administraciones del modelo neoliberal y sus tensiones.

\section{Método}

Utilizando un enfoque de investigación cualitativo, este trabajo apunta a la comprensión de las reinterpretaciones del Estado social a partir del análisis de tres fuentes: entrevistas semiestructuradas realizadas a tomadores de decisiones y consejeros y consejeras expertos en políticas sociales (que participan en los gobiernos entre 1973-2013); textos legales (leyes, decretos, normas) que regulan los sectores estudiados; y documentos oficiales del gobierno central del periodo descrito.

A través de un enfoque de análisis de políticas públicas, analizaremos los fenómenos desde las tensiones en juego y los encuentros. La revisión del proceso a partir de los registros normativos, los actores y el Estado, abre la posibilidad de visualizar las tensiones, así como las rupturas, en tanto manifestaciones de los campos en disputa en un momento determinado. En este sentido, el análisis de políticas públicas propone una descodificación de la historia institucional para captar sus lógicas estructurantes, lo que se traduce en términos de la realización de entrevistas en una reflexión sobre la relación entre el actor portador de representaciones y la institución en la que participa y de la que es objeto de su discurso (Bongrand \& Laborier, 2005). El objetivo es trazar las dinámicas de las reconfiguraciones del Estado social con el fin de interpretar sus movimientos, sus límites, pero, sobre todo, los procesos de conformación de las prácticas de lo social.

Esta investigación propone articular diferentes ámbitos para reconstruir la narrativa de la filosofía social del Chile neoliberal, para lo cual analizamos las posiciones de los actores involucrados (partidos y sectores políticos, expertos y think tanks), los cuerpos normativos del Estado social (especialmente las leyes que rigen la protección de la infancia y la construcción de lo social) y la lectura crítica de literatura especializada sobre la protección en Chile y sobre los enfoques estatales de lo social. La articulación de estas tres esferas nos llevará a un análisis de la complejidad del proceso de construcción múltiple del Estado social y de la filosofía de lo social contemporáneo en relación con: los actores, el proceso de articulación entre las esferas económica, política y social, y la naturaleza de las instituciones. 
Para hacer frente a este desafío, comenzamos por realizar un análisis de la bibliografía especializada sobre el Estado social en Chile y, en particular, de aquella oficial que describe las políticas sociales. Este trabajo basado en fuentes secundarias está estructurado en dos partes: la primera se refiere a la lectura de trabajos de historia social y documentos de los gobiernos y ministerios sociales del siglo XX, análisis que configura la hipótesis sobre la existencia de una estructura de acceso a los derechos, forjada desde 1833. La segunda parte recopila documentación oficial de los gobiernos estudiados ( 5 en total, entre 1973 y 2013), tales como balances anuales, programas gubernamentales, actas presidenciales, diagnósticos, informes y evaluaciones de los ámbitos de la protección social, la salud, la educación y la protección especializada.

La segunda fuente es la legislación de las áreas sociales entre 1973 y 2013, específicamente, 141 documentos legislativos (leyes orgánicas, leyes, decretos y normas). Estos textos pertenecen al sector social en general y a los sectores específicos de interés de la investigación.

La tercera fuente de análisis son 28 entrevistas semiestructuradas realizadas a:

- Responsables de la toma de decisiones que formaron parte de los Gobiernos entre 1973 y 2013 (Ministros/as, Directores/as de programas nacionales, entre otros).

- Expertos y expertas en las áreas analizadas que integraron los equipos ministeriales de alguno de los gobiernos estudiados o fueron asesores desde los think-tanks asociados a los gobiernos.

Las entrevistas duraron entre una hora y media y dos horas cada una; se realizaron con base en una guía de preguntas enviada con anticipación. Se realizaron procesos de consentimiento informado y los nombres de las personas fueron anonimizados. El trabajo de campo tuvo lugar en las ciudades de Santiago y Valparaíso, entre enero y mayo de 2015, y las entrevistas fueron realizadas en las oficinas de las personas entrevistadas.

El análisis de las tres fuentes se realiza mediante técnicas de análisis de contenido, que permite identificar y sistematizar tanto el contenido de los mensajes como la expresión de estos, con el fin de proponer deducciones lógicas y justificadas de las manifestaciones de la fuente y su contexto. La definición de las unidades de análisis se realizó a partir de declaraciones extraídas de las leyes y sentencias de los entrevistados, lo que permitió construir siete categorías de análisis vinculadas al tema en cuestión, los intereses de los actores y los principios evocados. 


\section{Hallazgos}

Podemos identificar como hallazgo principal de la investigación que el proceso neoliberal que experimenta Chile refleja, por una parte, la continuidad de una estructura desigual de distribución del acceso a derechos y, por otra, la introducción de tres inflexiones neoliberales que dan forma al nuevo Estado social, el que denominamos «Estado residual aumentado».

En una perspectiva histórica, los sistemas de protección y del Estado social en Chile resultan de un largo proceso de encuentros y desencuentros, entrelazados con la propia historia de la República, de 1810 a 1973. El análisis de estos procesos sociohistóricos en Chile nos llevará a la descripción de una formación basada en dos vías o redes de acceso a los derechos, caracterizada por dos rasgos: la centralidad del rol de la familia y la alianza público-privada.

Las políticas sociales del Estado chileno se desprenden del modelo de desarrollo configurado por las élites a partir del siglo XIX, y establecen la arquitectura de un sistema de distribución de la riqueza, la seguridad y las oportunidades, basado en la clasificación de la población según el grado de participación en el mercado laboral. Este sistema propone distintos canales de protección para los incluidos (tanto en términos socioeconómicos como políticos) y canales de asistencia para los exchidos. La estructura desigual del acceso a derechos dará lugar a la configuración de las instituciones de mediación entre el Estado, la familia y el mercado, así como a la concepción del bienestar en Chile.

Los procesos de transformación de lo social y del rol del Estado a los que asistimos en Chile a partir de 1973 proponen un nuevo proyecto en el horizonte normativo. El vínculo social de la matriz nacional-popular que precede a la matriz neoliberal-autoritaria (basada en un pacto de cooperación interclases) es remplazado por la concepción de la libertad individual y de la protección de los derechos sociales - ya fuertemente segmentada y desigual-y se verá confrontada a su reinvención a partir de dinámicas mercantiles. Este proceso se inscribe en una trayectoria histórica e interviene en la actualización de un relato de modernización, por lo que la preexistencia de una estructura histórica de distribución de derechos aparece como una condición de posibilidad para la reconfiguración del Estado social durante la dictadura (Cubillos-Celis et al., 2019).

La transformación neoliberal introducirá nuevos elementos a dicha estructura, manteniendo el esquema de desigualdades, los que describiremos como inflexiones: la primera 
se refiere a la mercantilización de los sectores sociales, es decir, a las reformas de modernización que liberalizan la oferta de servicios. La segunda inflexión analiza la introducción de la tecnocracia como racionalidad normativa de la toma de decisiones del sector social, donde el Estado asume un rol fuertemente regulador de la gestión en lo que se refiere a la transferencia de recursos, el control de la utilización de estos y la evaluación de resultados. Por último, la tercera inflexión se refiere a la transformación de la naturaleza de la focalización que, gracias a la multiplicación de criterios, opera como un dispositivo de justicia social residual en el marco de profundas desigualdades provocadas por el cambio de matriz socioeconómica. El gasto social se orienta hacia un modelo no-distributivo de acceso a derechos.

En este contexto, la matriz neoliberal chilena se presenta como un modelo híbrido que se configura a partir de los rasgos de la estructura histórica de distribución desigual del acceso a derechos sociales y de las inflexiones introducidas durante el régimen militar; aspecto que se evidencia en la protección de la infancia a través de los sectores de la salud, la educación y la protección especializada.

Las reformas efectuadas no solo aportarán cambios administrativos a la prestación de servicios, sino que procederán a una metamorfosis del rol histórico del Estado, instalando cifras negras a nivel social: en 1988 la tasa de pobreza se situaba en $26.8 \%$ y la tasa de indigencia alcanzaba el $22.9 \%$, es decir, un total de $49.6 \%$ de pobreza absoluta. La población infantil se vio especialmente afectada por dicho fenómeno: en 1990, $52.3 \%$ de los niños y niñas entre o y 9 años se encontraban bajo la línea de la pobreza. ${ }^{3}$ La liberalización del mercado, las crisis económicas (1975 y 1982) y la flexibilización del trabajo, caracterizarán la reconfiguración del Estado social realizada por la alianza cívico-militar en el poder y por los Chicago Boys, ${ }^{4}$ estableciendo un nuevo marco de relaciones entre el Estado, el mercado, las familias y los individuos. Este proceso tomará forma en las áreas analizadas, a partir de la introducción de las inflexiones mencionadas, que analizamos a continuación.

\footnotetext{
${ }^{3}$ Los datos extraídos de la Encuesta de caracterización socioeconómica nacional, que se aplica desde 1985. Se utilizado el concepto de pobreza absoluta medida a partir del método de ingresos, es decir, de necesidades básicas insatisfechas en relación con una canasta básica de alimentos.

${ }^{4}$ Grupo de economistas formados en la Escuela de Chicago, Estado Unidos, entre 1956 y 1970, que propondrá medidas para la transformación del modelo chileno. Su propuesta, «El Ladrillo», será entregada a la Junta Militar el día después del golpe (12/o9/1973), saliendo a la luz pública en 1992 editada por el Centro de Estudios Públicos.
} 


\section{Liberalización de la oferta de servicios}

Las reformas de desconcentración de los servicios públicos, de descentralización de competencias hacia las colectividades territoriales y de privatización de la provisión de servicios introducirán mecanismos de mercado y de cuasimercado al sector social, transformando la concepción del ejercicio de derechos.

En el sector salud, se disuelve el Sistema Nacional de Salud, los actores se diversifican y los antiguos proveedores se transforman. El fondo colector universal se divide en dos subsistemas: el Fondo Nacional de Salud y las Instituciones privadas de Salud Previsional. El principio de libre elección y la descentralización convierten al sistema de salud en un sector competitivo, gracias a la introducción del financiamiento por prestaciones a la oferta y la demanda, así como a la diversificación de los prestadores de servicios que incluirán al sector público, al sector privado y a las corporaciones municipales de salud y educación (instancias de carácter privado que administrarán los centros municipales de atención primaria). Con este diseño se introducen «diferentes tratos en la atención, que varían en función de si el beneficiario pertenece al sistema público o privado, y de si se atiende en el sector público o privado», nos explica Jaime, jefe de una división del Ministerio de Salud entre 2006 y 2010.

En el sector de la educación, la reforma se apoyará en la desconcentración de competencias, pasando de servicios estatales a servicios regionales y luego comunales (decreto 1586, decreto de ley 824 de 1974, decreto 721 de 1977), con el objetivo de maximizar la eficiencia en la utilización de los recursos, introduciendo la transferencia de funciones, la competencia entre los establecimientos (Cox, 2003) y decretando las mismas normas de financiamiento a la educación municipal que a la privada, a través del subsidio a la demanda (matrícula/asistencia escolar diaria). El sistema de educación primaria se convertirá entonces en un modelo mixto conformado por un conjunto de establecimientos de gestión municipal, particulares a cargo de los sostenedores con subvención municipal y establecimientos privados dirigido a las élites ( $8 \%$ de la matrícula). En 1989, a fines del régimen, $58.6 \%$ de la matrícula de la educación primaria se concentrará en los establecimientos municipales y el $34 \%$ habrá emigrado hacia el sistema particular-subvencionado; al año 2010, el sector municipal desciende al $40 \%$, mientras el sector particular-subvencionado aumenta al 50\% (Ministerio de Educación Pública, 2011).

El sistema permitirá la creación de un mercado de la educación, que llegará a su corolario con la Ley de Financiamiento Compartido, DFL 5 (Ministerio de Educación Pública, 1993), la que introduce el copago de las familias en los establecimientos con subvención, 
con el objetivo de dinamizar la competencia y el lucro. Según Pedro, exdirector de la División de Presupuesto del Ministerio de Educación durante los 9o y consejero del presidente Eduardo Frei (1994-2000), «la reforma inicial del sistema de subsidios tuvo muchos problemas de formulación y fue muy simplista. Todo había sido hecho por los generalistas para quienes todo se resuelve introduciendo un mercado simplificado sin intervención». La Ley de Financiamiento Compartido habría permitido corregir estos aspectos, cambiando para siempre el rostro de la educación. Lo público será asociado a servicios hacia los pobres, mientras que el sector privado se convertirá en un motor del mercado.

Por último, en el sector de la protección especializada, en 1979 se crea el Servicio Nacional de Menores (Sename), entidad estatal alojada en el Ministerio de Justicia, que tiene por objetivo atender la población infantil en situación de riesgo. El Decreto 356 establece que el Sename es un órgano central que regulará el funcionamiento del sistema, introduciendo una nueva lógica en la relación entre las instituciones privadas y el Estado, a través de la subvención por niño/a, y una lógica de oferta y demanda, según un esquema de economía competitiva que no se centra en la lógica del bienestar sino en la atención masiva (Álvarez, 1993). El Sename opera con una lectura de pobreza como factor de riesgo. Según Carry, experta del Consejo Nacional de la Infancia (2010-2013), «la dictadura subvencionará a un conjunto de actores privados que atienden a los niños en situaciones precarias».

En conclusión, los tres sectores observados son objeto de reformas que transforman, junto con los mecanismos de financiamiento, el rol social del Estado, replegándose a un rol de subsidio y regulador de mercado, consolidando progresivamente el mercado de bienes sociales.

\section{La tecnocracia como racionalidad normativa de la}

\section{toma de decisiones}

Las reformas económicas y sociales generarán una precarización masiva las condiciones de vida, y en 1990 el umbral de pobreza supera el $51.2 \%$ de la población. En el nuevo modelo la pobreza se considera un daño colateral, bajo el supuesto de que, a través del crecimiento económico, los beneficios del progreso beneficiarán paulatinamente a toda la población (teoría del chorreo)5. En dicha lógica de naturalización de los efectos del

5 Teoría Trickle down effect de la economía neoclásica asociada a Escuela de Economía de Chicago, liderada por Stigler y Friedman. 
mercado, el gobierno militar adoptará medidas para combatir la pobreza, que es percibida como un freno al modelo y un obstáculo al desarrollo.

Para ello, uno de los primeros retos es conocer las características de esta nueva pobreza resultante del modelo en experimentación. La construcción de múltiples baterías de medición se convierte en prioridad: el Mapa de la Extrema Pobreza (1975), la Encuesta de Características Socioeconómicas (1982) y la Ficha de los Comités de Asistencia Social (1980) son herramientas que permitirán al régimen tecnificar la política social (Larrañaga, 2010). En adelante, la política social se guiará por indicadores, categorías socioeconómicas y clasificaciones estadísticas que determinan la posición de las familias. El propósito es identificar a la población potencialmente beneficiaria de los programas y observar los resultados, lo que requerirá de personal con altas competencias técnicas, tanto a nivel central como en el terreno.

Esta configuración de las políticas sociales marca la estrategia de intervención social que subyace a la política social de la dictadura y luego de los gobiernos democráticos. La diversificación de los instrumentos de clasificación, selección y seguimiento de las personas beneficiadas encuentra una fuente de desarrollo en la filosofía de crecimiento y equidad que acompaña la transición democrática (1990-1999) y luego en la concepción de crecimiento con igualdad postulada durante el periodo de consolidación (2000-2013). El paso a la gestión de licitaciones de recursos públicos encuentra un corelato en el proceso de tecnificación de las intervenciones sociales y, de la misma manera, dará paso paulatino de un lenguaje de la asistencia al de gestión.

En este marco, se establecerán mecanismos específicos para la clasificación de la infancia en todos los campos de intervención, a través de una fórmula que combina tradición e innovación. En términos de la salud, la tecnocratización se manifiesta en la necesidad de identificar las poblaciones beneficiarias, y combina los programas de seguimiento maternoinfantil inaugurados por el gobierno de Allende con prestaciones específicas de nutrición durante la dictadura, ampliación de la vacunación y prestaciones en transición. Finalmente, el sistema de protección integral a la primera infancia Chile Crece Contigo (2010) (ChCC), el que fue posible gracias a la continuidad del proceso: «Un insumo potente que facilitó el desarrollo (...) del ChCC fue la creciente "tecnificación" de la política social que tuvo el país desde la década de 1980 hasta el presente» (Valderrama \& Raczynski, 2010, p. 9).

En el área de la educación, el proceso es similar respecto a la tecnificación de los criterios de financiamiento por matrícula y asistencia, además de un proceso paulatino de 
subvenciones proporcionales por comuna. A partir de la transición, el principio de calidad provocará la proliferación de instrumentos de seguimiento, medición y resultados, que se traducirá en una multiplicidad de programas diferenciados por territorio y población (especialmente el P-goo y los programas Mece). Durante el periodo de consolidación democrática, el eje de calidad ya institucionalizado, se introducirán diversas medidas de discriminación positiva a través la Ley General de Educación, 20370 (Ministerio de Educación Pública, 2009) y programas dirigidos al estudiantado en situación de vulnerabilidad, en el marco de la Ley de Subvención Escolar Preferencial 20248 (Ministerio de Educación Pública, 2008), a la vez que se otorga prioridad a la primera infancia ampliando los servicios. A partir de esta última la ley se incorporan nuevos actores privados encargados de la asistencia técnica a las escuelas, quiénes abrirán un nuevo mercado de servicios.

Por último, en el área de la protección especializada se observa la necesidad de justificar cuántos niños y niñas son atendidos por los programas, cómo y con qué propósito, estableciendo criterios cuantitativos de intervención, a partir de indicadores de acciones directas e indirectas. Los fondos de financiamiento se licitarán periódicamente en función del logro de los objetivos y resultados. Estos procesos afectarán directamente el significado y calidad de la intervención social, creando «una procedimentalización del trabajo, una desafección de la intervención, así como una sensación de baja efectividad», explica Carla, experta en protección especializada. La lógica tecnocrática fortalecerá las grandes asociaciones con mayor capacidad de gestión y poder de negociación, según explica Orlando, consejero en protección especializada de los gobiernos de 1990-2000.

Finalmente, aunque la tecnificación tomará forma en términos de regulación central, este camino no estará exento de resistencias, y expresará dos puntos críticos de la inflexión neoliberal: la imposición de prácticas estandarizadas en el ámbito administrativo -que cohabitarán con las prácticas tradicionales-y planteará interrogantes debido a los procesos de opacidad de la complejidad de los fenómenos sociales resultado de la simplificación a través de cifras e indicadores.

\section{La focalización ampliada como mecanismo de justicia social residual}

El papel subsidiario del Estado se traduce en la focalización de la pobreza y la distribución del gasto social hacia la infancia, como ejes que buscan evitar la deserción social 
potencial de una parte población e invertir en recursos humanos con vistas a una autonomía de estos en el mercado.

En el sector de la salud, el Estado conservará ciertas competencias respecto a la cobertura de las poblaciones en situación de pobreza y de la infancia. El régimen militar otorgará alta prioridad a la lucha contra la desnutrición infantil, que en 1973 causa el 25.3\% de mortalidad neonatal (Instituto Nacional de Estadísticas, 2011). Así, la reducción de la mortalidad infantil se convertirá en una oportunidad para legitimar el modelo. Germán, consejero de la Junta Militar en los temas sociales y arquitecto de las políticas sanitarias de la infancia, afirma: «Empezábamos a dar la impresión de que no sabíamos [la Junta Militar] para donde ir; necesitábamos un indicador, y la mortalidad infantil es un indicador fiable del desarrollo; la estrategia de nutrición fue ascendente y tuvo excelente recepción en la comunidad».

Así, se formularán políticas centrales de vacunación, programas nacionales de nutrición y el programa Control del Niño Sano. El sector privado filantrópico jugará un rol fundamental en la implementación de programas de alimentación para población infantil de riesgo, mientras que los mundos asociativo y universitario participarán a través de la recolección de fondos y de la investigación. En 1989, la tasa de mortalidad infantil descenderá a $9 \%$ y el éxito de dicha estrategia focalizada se cristalizará en la continuidad de los programas durante el periodo de transición. Sin embargo, durante el periodo de consolidación democrática, Chile Crece Contigo (Ministerio de Planificación) propondrá un sistema de prestaciones basadas en indicadores de desarrollo infantil, dirigidas al conjunto del sistema público y nuevas prestaciones serán focalizadas por criterios de vulnerabilidad. Así, la salud pública infantil se reformula desde una concepción mixta de acceso a servicios y de focalización de prestaciones especializadas.

En el sector de la educación, la focalización del gasto en escuelas municipales disminuirá la matrícula del sector público, dando paso a lo que Julio, responsable de educación primaria del gobierno del presidente Patricio Aylwin (1990-1994), describe como «un concepto de Estado subsidiario que se encarga de aquellos que quedan atrás en el área de la educación primaria e inicial». Por ello, uno de los desafíos de la transición será abordar la llamada «deuda social de la dictadura» y, junto con profundizar las medidas de focalización, se orientará la inversión a la calidad de la educación con el fin de generar mecanismos correctores. Así, observamos que tanto en los programas de equidad del período de transición, como en los de igualdad de oportunidades de la década 2000-2010, 
el desarrollo de la educación está centrado en el mejoramiento del acceso y de la calidad de la educación dirigida a los sectores más pobres.

En la educación inicial observamos la misma dinámica, favoreciendo principalmente el aumento de la cobertura del sector público (dirigido a niños y niñas de los quintiles más pobres), administrado por la Junta Nacional de Jardines Infantiles (Junji) dependiente del Ministerio, e Integra (fundación dependiente de la Presidencia). El aumento más importante de la cobertura se realiza entre 2006 y 2009 en el marco del componente focalizado del ChCC, superando el 500 \% bajo la tutela de Junji (Chile Crece Contigo, 2010).

En lo que se refiere a la protección especializada, la focalización opera a través del criterio del riesgo social, asociado a la insatisfacción de necesidades básicas. La inversión para implementar el Sename es importante en un inicio, pero los montos de subvenciones disminuirán a menos de la mitad durante la década de los ochenta. La precariedad del sistema impone una lógica de supervivencia a las instituciones, las cuales van a centrar sus criterios de intervención en ámbitos no especializados que refuerzan el carácter residual de la protección dirigida a los pobres. «Cuando había un niño sano que ya estaba acostumbrado a las reglas del juego, el objetivo era tratar de mantenerlo hasta los 18 años», dice Franck, especialista y antiguo director de desarrollo social de un organismo internacional.

La intervención del Estado en esta materia se afirma en la doctrina de la irregularidad social, donde la infancia en dificultad o los denominados «menores», constituyen un riesgo para la estabilidad social (García-Méndez, 1997). Dicha concepción entrará en tensión con la lógica neoliberal tecnócrata y la ratificación de la Convención Internacional de los Derechos de la Infancia y la Adolescencia (1990) que introducirá la perspectiva de derechos, pondrá aún más en tensión la lógica de intervención residual, obligando a repensar la protección desde la vulneración de derechos y no desde la pobreza.

En resumen, un debate de larga data cuestiona la efectividad de las políticas sociales focalizadas, existiendo ciertos acuerdos tanto en torno a la eficacia transitoria de los resultados, como a los problemas ético-normativos que esta perspectiva impone. Desde la definición de las personas que serán foco de los programas hasta la realización de los objetivos, la focalización requiere de fuertes capacidades técnicas y de amplios consensos políticos. Sin embargo, el riesgo mayor es que a través de los mecanismos de justicia reparativa se instalan condiciones para la profundización de desigualdades. El caso chileno, en efecto, es reconocido por los factores de segregación social encabezando las cifras de desigualdad en la región. 


\section{Síntesis}

Las preguntas que surgen luego de la revisión de las tres grandes inflexiones neoliberales se apuntan fundamentalmente al tipo de sociedad que se construye bajo los diversos paradigmas de la protección y al rol juegan los Estados frente a los derechos sociales en un contexto de neoliberalización creciente de las economías mundiales. El desafío supera, de esta forma, los aspectos vinculados al diseño de la política social, para trasladar la pregunta sobre la construcción de lo social y de lo político en las sociedades contemporáneas, en tanto disputa por las concepciones de distribución de lo común. Una reflexión en torno a dichas preguntas y desafíos se presenta a continuación.

\section{Discusión}

Los procesos analizados se desarrollan progresivamente durante los 40 años de formación y adaptación de las dinámicas neoliberales, encontrando resistencias y críticas en su trayectoria, sin que ello impida su desarrollo. Sin embargo, desde una perspectiva sociológica, las transformaciones que operan en el marco de las políticas sociales de la infancia darán cuenta de la configuración de lo social y lo político propia de un modelo de desarrollo sin precedentes.

La simbiosis del sector social con las dinámicas del mercado constituye, en fin, el vector de la implementación del neoliberalismo en las diferentes esferas de la vida social y la consagración de la filosofía del Estado residual aumentado. Así, la colonización de lo social y la introducción de la competencia generalizada a la base del vínculo social contemporáneo (a través del mérito, el éxito escolar y profesional, la libre elección de servicios, entre otros) cristalizarán el cambio de paradigma políticoeconómico de la nueva matriz. La centralidad articuladora del mercado y la capacidad reguladora del Estado se enraízan en la vida cotidiana a través de los sistemas de acceso a derechos y modelan la familia a través de la intervención infantil.

Esta concepción sociopolítica replanteará las bases del vínculo social reflejando tres grandes tensiones: la crisis normativa del pacto de cohesión social, la naturalización y legitimación de las desigualdades y la despolitización de los derechos sociales.

En primer lugar, la crisis normativa del pacto de cohesión social hace referencia a los rasgos que sostienen las relaciones entre individuos e instituciones, y a la concepción de seguridad en una sociedad determinada. El modelo de políticas sociales implementado 
entre 1973 y 2013 concibe la protección a través de la operacionalización de la noción de bienes y servicios que se obtienen por la vía de la integración económica. Quienes no tengan éxito en dicha integración y, por ende, no desarrollen las posibilidades de garantizar su protección, serán definidos como poblaciones vulnerables, a quienes el Estado deberá asistir, en una primera etapa desde una perspectiva residual-subsidiaria simple, luego a través de las políticas de lucha contra la pobreza y, finalmente, a través del llamado sistema de protección social. En una perspectiva de identificación y de focalización de dichas poblaciones insuficientemente integradas al mercado, la protección social actúa como un mecanismo diferenciador y delimita la intervención.

Esta concepción de la protección social vinculada a la vulnerabilidad selectiva, hará eco en la emergencia del fenómeno de inconsistencia posicional (Araujo \& Martuccelli, 2011) que experimenta la sociedad chilena y que determina la exposición de los individuos frente a las fragilidades de una posición social que depende de un contexto inestable (marcado por la mediación del mercado) y ausente de protección. La inconsistencia posicional cristaliza los miedos de las múltiples fuentes de vulnerabilidad del Chile neoliberal: el miedo a la movilidad descendiente, a enfermarse, a la posibilidad de no ser integrado al mercado laboral o ser expulsado de este, fragilizando así la cohesión social y la sensación de seguridad. La infancia se desarrolla en este contexto de competencia e incertidumbre, donde la escuela deja de ser un espacio de integración para devenir un espacio de diferenciación.

Este aspecto se vincula directamente con la segunda de las tensiones identificadas: la naturalización y legitimación de las desigualdades. Paulatinamente, desde 1990, la acción del Estado social se desplaza desde la sobrevivencia absoluta de poblaciones en dificultad, hacia las dificultades de integración al mercado. En esta concepción se funda un Estado que supone una política social integrada a los intereses de la política económica, que direcciona la intervención pública hacia el diseño e implementación de mecanismos de facilitación de la integración al mercado. La acción focalizada en las poblaciones con integración deficiente provoca que la política de igualdad de oportunidades se convierta en una operación de justicia social que legitima las desigualdades en el acceso a los derechos, disociando al Estado de acciones redistributivas (Rosanvallon, 2011).

La inversión en políticas de salud y educación para la infancia en situación de vulnerabilidad social tiene por objetivo eliminar las desigualdades de inicio de trayectoria de vida (Chile Crece Contigo, 2010). Sin embargo, esta filosofía representa un proyecto de igualdad estadística y no de igualdad sustancial de derechos (Rosanvallon, 2011), ya que una vez sobrepasado el límite de la definición de la condición de pobreza económica, los 
individuos son abandonados al mercado, fragilizando su posición. En este sentido, el objetivo de este modelo de bienestar apunta a reparar las deficiencias de la esfera económica en una lógica indemnizatoria, otorgando al Estado un rol fundamental en el plano de la distribución de las oportunidades de integración al mercado (Esping-Anderson \& Palier, 2008). El Estado de carácter residual extiende así su campo de acción subsidiario para ejercer un rol de coordinador y fiscalizador de las diversas esferas donde invierte socialmente, con el objetivo de evitar una ruptura del vínculo de la población más frágil en términos de integración y, por ende, con capacidad competitiva más baja. Dicha noción del bienestar se legitima como mecanismo de justicia social en un contexto de desigualdades estructurales, emergiendo en un contexto de alta competitividad donde los vínculos sociales tradicionales de protección, asociados a la familia, la escuela o el trabajo, son invadidos también por una lógica mercantil.

En este escenario observamos la tercera tensión: los derechos sociales son banalizados y convertidos en bienes sociales, favoreciendo su despolitización. Esto debido a que la focalización de la intervención social y la creación de un mercado de prestaciones implica la exclusión explícita de ciertas categorías de población del acceso a la protección de derechos (como la salud o la educación), lo que conlleva tanto el riesgo de exclusión simbólica como de negación del acceso a los bienes primarios (Rawls, 2009). Así, por una parte, la categorización del acceso a bienes y servicios conduce a procesos de descalificación social (Paugam, 2012) que se traducen en la configuración de un estatus social desvalorizado, dificultando las relaciones con el otro social y estigmatizando la posición social de la persona focalizada. Por otra parte, reconfigura la adquisición de servicios a través del mercado, como símbolo de éxito, de integración, de mérito o de privilegio.

Los derechos sociales como base universal son reconceptualizados, siendo despojados de su carácter universal y la justicia reparativa instala las condiciones para la profundización de las desigualdades. El caso chileno, en efecto, encabeza las cifras de desigualdad de los países pertenecientes a la Organización para la Cooperación y el Desarrollo Económico (2015).

En conclusión, la existencia de estas tres tensiones del Estado residual aumentado nos da cuenta de los desafíos que impone este tipo de modelos de distribución de acceso a derechos a la construcción de las sociedades y al pacto social. La infancia se posiciona en un terreno de transformación, no solo de dispositivos de gestión, sino que se convierte en actor principal de un proceso de experimentación social, planteando una serie de desafíos a la noción misma de derechos y a las dinámicas de protección de estos. 


\section{Consideraciones finales}

Las transformaciones experimentadas por el Estado social chileno desde 1973 son incontestables; sin embargo, como pudimos observar, los cambios se posicionan en una línea de continuidades respecto a una estructura de distribución desigual del acceso a derechos y en una institucionalidad de larga data.

Los diversos aspectos que intervienen en el cambio de carácter del Estado social desde la dictadura - y que serán consolidados durante el periodo democrático- observados en las políticas sociales de protección de derechos de la infancia analizadas, operarán como dispositivos de corrección de la capacidad de competencia de los individuos para insertarse en el mercado y generar, de tal manera, procesos de integración social. Desde esta perspectiva, la protección social y las políticas de acceso a derechos fundamentales durante la infancia se convierten en espacios de igualación de capacidades. Esta fórmula legitima las desigualdades estructurales, aceptando las condiciones que las originan y bajo el supuesto de corrección de los factores de desequilibrio.

El desafío supera, de esta forma, los aspectos vinculados al diseño de la política social, para trasladar la pregunta sobre la construcción de lo social y de lo político en las sociedades contemporáneas, en tanto campo de disputa por las concepciones de distribución de lo común, situando a la infancia como el espacio de modelización del nuevo pacto social. El Chile neoliberal impone así un debate necesario respecto al rol de la infancia frente a las sociedades en transformación y a la necesidad de repensar la protección en términos técnicos, normativos y filosóficos.

\section{Referencias}

Álvarez, J. (1993). La experiencia neoliberal en la atención de menores en riesgo social en infancia en riego social y políticas sociales en Chile. Instituto Interamericano del Niño, Servicio Nacional de Menores.

Araujo, K., \& Martuccelli, D. (2011). La inconsistencia posicional: un nuevo concepto sobre la estratificación social. Revista Cepal, (103), 165-178.

Bongrand, P., \& Laborier, P. (2005). L'entretien dans l'analyse des politiques publiques: un impensé méthodologique? Revue Française de Science Politique, 1(1), 73-111. https:// doi.org/10.3917/rfsp. 551.0073 
Cox, C. (2003). Las políticas educacionales de Chile en las últimas dos décadas del siglo XX. Editorial Universitaria.

Cubillos-Celis, P. (2019a). Neoliberalismo, focalización e infancia en Chile: 1973-2010. Revista Mexicana de Sociología, 81(3), 611-636.

Cubillos-Celis, P. (2019b). Enfance et néolibéralisme au Chili. Chroniques d'une Dépossession. Editions L'Hartman.

Cubillos-Celis, P., Slachevsky, N., \& Yañez, R. (2019). El Estado social de mañana: diálogos sobre bienestar, democracia y capitalismo, con Göran Therborn, Philippe Van Parijs y Nicolas Duvoux. Lom.

Chile Crece Contigo. (2010). Cuatro años creciendo juntos. Memoria de la instalación del Sistema de Protección Integral a la Infancia Chile Crece Contigo, 2006-2010. Autor.

Dardot, P., \& Laval, C. (2010). La nouvelle raison du monde. Essai sur la société néolibérale. Éditions La Découverte.

Esping-Andersen, G. (2009). Les trois mondes de l'État-providence. Essai sur le capitalisme moderne. PUF.

Esping-Andersen, G., \& Palier, B. (2008). Trois leçons sur l'État-providence. Editions du Seuil. Filgueira, F. (2007). Cohesión, riesgo y arquitectura de protección social en América Latina. Cepal.

García-Méndez, E. (1997). La Convención internacional de los derechos del niño: del menor como objeto de la compasión-represión a la infancia-adolescencia como sujeto de derechos. Forum Pacis.

Gough, I., \& Therborn, G. (2010). The global future of welfare states. En F. G. Castles, S. Leibfried, J. Lewis, H. Obinger, \& C. Pierson (Eds.), The Oxford handbook of the welfare state. Oxford University Press https://doi.org/10.1093/oxfordhb/9780199579396.003.0048

Harvey, D. (2005). A brief history of neoliberalism. Oxford University Press.

Hayek, F. (2014). La route de la servitude. PUF.

Instituto Nacional de Estadísticas. (2011). Estadísticas vitales, Informe anual 2009. Autor.

Larrañaga, O. (2010). El Estado de Bienestar en Chile: 1910-2010. En R. Lagos (Ed.), Cien años de luces y sombras. Taurus.

Ministerio de Educación Pública. (1993). DFL 5. Fija texto refundido, coordinado y sistematizado del decreto con fuerza de ley 2, de 1989, sobre subvención del Estado a establecimientos educacionales. Autor.

Ministerio de Educación Pública. (2008). Ley 20 248. Establece la Ley de Subvención Escolar Preferencial. Autor. 
Ministerio de Educación Pública. (2009). Ley 20 37o. Establece la Ley General de Educación. Autor.

Ministerio de Educación Pública. (2011). Estadísticas de la Educación, 2011. Autor.

Ministerio de Planificación. (2009). Ley 20 379. Crea el Sistema Intersectorial de Protección Social e institucionaliza el Subsistema de Protección Integral a la Infancia Chile Crece Contigo. Autor.

Organización para la Cooperación y el Desarrollo Económico. (2015). Estudios económicos de la OCDE, Chile. Autor.

Paugam, S. (2012). Les formes contemporaines de la disqualification sociale. CERISCOPE Pauvreté. http://ceriscope.sciences-po.fr/pauvrete/content/part5/les-formescontemporaines-de-la-disqualification-sociale

Rawls, J. (2009). Théorie de la Justice. Points.

Rosanvallon, P. (2011). La société des égaux. Editions du Seuil.

Titmuss, R. (1974). Social policy. Open University Set Book.

Valderrama, C., \& Raczynski, D. (2010). Chile Crece Contigo: un caso particular de scaling up. Asesorías para el Desarrollo. 